\title{
Generalized Hantzsche-Wendt flat manifolds
}

\section{Juan P. Rossetti and Andrzej Szczepański}

\begin{abstract}
We study the family of closed Riemannian $n$-manifolds with holonomy group isomorphic to $\mathbb{Z}_{2}^{n-1}$, which we call generalized HantzscheWendt manifolds. We prove results on their structure, compute some invariants, and find relations between them, illustrated in a graph connecting the family.
\end{abstract}

\section{Introduction}

A flat manifold is a closed Riemannian manifold with zero sectional curvature. From Bieberbach theorems, it is known that in each dimension there is only a finite number of such manifolds, up to affine equivalence, and the problem of classifying them is important. Recently, this has been achieved up to dimension six (cf. [3]), but it is not possible to do it in general. Hence some interesting families of flat manifolds have been considered, as for instance those with first Betti number zero or those with holonomy group $\mathbb{Z}_{2} \oplus \mathbb{Z}_{2}$.

The purpose of this article is to study another special class of flat manifolds, namely those of dimension $n$ with holonomy group isomorphic to $\mathbb{Z}_{2}^{n-1}$. In dimension two the Klein bottle belongs to this family and in dimension three there are three such manifolds: a classical flat manifold first considered by Hantzsche and Wendt [7] (now called didicosm, see last section) and two non-orientable ones.

Though here they are considered for first time together, these manifolds have been studied partially in [12], [13], [14], [15], [17], mainly in the oriented case, where we shall call them Hantzsche-Wendt manifolds (HW manifolds for short; respectively HW (Bieberbach) groups, if we consider their

2000 Mathematics Subject Classification: Primary: 20H15, 57S30. Secondary: 53C29, 20F $34,57 \mathrm{~N} 16,05 \mathrm{C} 25$.

Keywords: Flat manifold, Bieberbach group, holonomy representation. 
fundamental groups, which are Bieberbach groups). They have many interesting properties, for instance, there are pairs of isospectral HW manifolds non homeomorphic to each other (cf. [14]). Moreover, HW manifolds have the $\mathbb{Q}$-homology of spheres (cf. [17]) and hence they are geometrically formal (cf. [11]). We shall call the manifolds in our family generalized Hantzsche-Wendt manifolds (GHW manifolds for short; respectively GHW (Bieberbach) groups).

One of the main results in this article is that the integral holonomy representation of a GHW manifold is of diagonal type (Theorem 3.1). This simplifies the study of this family. An immediate consequence is that the HW manifolds are exactly those considered in [14].

Another interesting property of these manifolds, or their fundamental groups, is given by the relations between them in different dimensions. In fact we show that every GHW group has a subgroup isomorphic to a GHW group of one lower dimension (Corollary 3.4). Moreover, from Theorem 5.1, any $n$-dimensional GHW group is a subgroup of some $(n+1)$-dimensional one. With these observations we define a graph, whose vertices correspond to GHW groups, connecting the family, which we draw up to dimension four in the last section.

It is clear that the number of non-oriented GHW flat manifolds is much greater than the number of oriented ones (see for instance the table in Section 4). In the non-oriented case, there are two types of GHW groups, depending on whether the first Betti number equals one or zero. We show that the case with first Betti number one (as well as the oriented case) has only one holonomy representation, which is completely determined. Hence it makes sense to carry out the calculation of the $\mathbb{Q}$-homology (see Theorem 4.2).

The situation in the non-oriented case with first Betti number zero is much more complicated since the holonomy representation is not unique. We find the exact number of integral representations occurring in this subclass and show that it grows linearly with the dimension. This follows as a consequence of Theorem 5.1, which asserts that every diagonal integral representation can be realized as the integral holonomy representations of a flat manifold. In Section 6 we consider some examples in this subclass which are similar to the HW manifolds from [17]. In Section 7 we study some symmetries of GHW manifolds. In particular, we determine the outer automorphism groups of certain subfamily of GHW groups with non-trivial center. Moreover a general upper bound of the order of the outer automorphism group of GHW groups is given.

There are some problems and questions which we formulate at the end.

We would like to thank Wim Malfait for discussions regarding Section 7. 


\section{Preliminaries and notations}

Let $\Gamma$ be a Bieberbach group of dimension or rank $n$. By definition it is a torsion-free, discrete, cocompact subgroup of the group $\mathrm{E}(n)=\mathrm{O}(n) \ltimes \mathbb{R}^{n}$ of rigid motions of the $n$-dimensional Euclidean space. The multiplication in $\mathrm{E}(n)$ is given by

$$
(B, b)(C, c)=(B C, B c+b),
$$

where $B, C \in \mathrm{O}(n)$ and $b, c \in \mathbb{R}^{n}$. The translation part $c$ of an isometry $(C, c) \in \mathrm{E}(n)$ shall sometimes be denoted by $s(C)$. If a flat manifold $\mathbb{R}^{n} / \Gamma$ is oriented then by definition $\Gamma$ is oriented. From Bieberbach theorems (cf. [2]), the translation subgroup $\Lambda \simeq \mathbb{Z}^{n}$ of $\Gamma$ is normal, maximal abelian, of finite index. Then there is a short exact sequence of groups

$$
0 \longrightarrow \Lambda \longrightarrow \Gamma \longrightarrow G \longrightarrow 0,
$$

where $G$ is finite and isomorphic to the holonomy group of the manifold $\mathbb{R}^{n} / \Gamma$, thus we call it the holonomy group of $\Gamma$. It is well known that any extension as (2.1) defines an element $\alpha \in H^{2}(G, \Lambda)$, where the $G$-module structure of $\Lambda$ is defined by conjugation in (2.1). We denote by $\rho$ this integral representation and call it the integral holonomy representation of $\Gamma$ (or of $\mathbb{R}^{n} / \Gamma$ ).

Definition 2.1 Let $\Gamma$ be a Bieberbach group of dimension $n$. A flat manifold $\mathbb{R}^{n} / \Gamma$ is called generalized Hantzsche-Wendt manifold if its holonomy group is $\mathbb{Z}_{2}^{n-1}$. In this case $\Gamma$ is called a generalized Hantzsche-Wendt group.

Definition 2.2 We say that a Bieberbach group $\Gamma$ is diagonal or of diagonal type if its corresponding lattice of translations $\Lambda$ has an orthogonal basis in which the integral holonomy representation $\rho$ diagonalizes. We will also call diagonal the corresponding flat manifold $\mathbb{R}^{n} / \Gamma$.

Remark 2.3 In [16], the previous definition was done with the (slightly more restrictive) assumption that the basis of $\Lambda$ is orthonormal. We recall that diagonal manifolds have necessarily the holonomy group isomorphic to $\mathbb{Z}_{2}^{k}$ for some $k \in \mathbb{N}$.

Consider

$$
C_{i}:=\left[\begin{array}{cccccccc}
1 & 0 & \cdots & 0 & 0 & 0 & \cdots & 0 \\
0 & 1 & \cdots & 0 & 0 & 0 & \cdots & 0 \\
\vdots & \vdots & \ddots & & \vdots & \vdots & & \vdots \\
0 & 0 & & 1 & 0 & 0 & \cdots & 0 \\
0 & 0 & \cdots & 0 & -1 & 0 & \cdots & 0 \\
0 & 0 & \cdots & 0 & 0 & 1 & & 0 \\
\vdots & \vdots & & \vdots & \vdots & & \ddots & \vdots \\
0 & 0 & \cdots & 0 & 0 & 0 & \cdots & 1
\end{array}\right], \quad 1 \leq i \leq n
$$


where the -1 is placed in the $(i, i)$ entry. For brevity we will denote $C_{i}=$ $\operatorname{diag}(1, \ldots, 1, \underbrace{-1}_{i}, 1, \ldots, 1)$, for $1 \leq i \leq n$. The product of two of these is

$$
C_{i} C_{j}=\operatorname{diag}(1, \ldots, 1, \underbrace{-1}_{i}, 1, \ldots, 1, \underbrace{-1}_{j}, 1, \ldots, 1), \text { for every } i<j .
$$

The elements in (2.3) generate the group $\mathrm{D}^{+}(n):=\mathrm{D}(n) \cap \mathrm{SL}(n, \mathbb{Z})$, of cardinality $2^{n-1}$, and of index two in the group $\mathrm{D}(n)$ of diagonal $n \times n$ matrices with entries \pm 1 in the diagonal.

In the oriented case with holonomy group $\mathbb{Z}_{2}^{n-1}$, the rationalization of the integral holonomy representation $\rho$ (i.e. to consider $\Lambda$ as a $\mathbb{Q}$-module instead of a $\mathbb{Z}$-module), gives a unique representation $\rho_{\mathbb{Q}}$ whose image, in an appropriate basis, is exactly $\mathrm{D}^{+}(n)$. Hence, when $n$ is even there are no HW manifolds, since $\mathrm{D}^{+}(n)$ contains $-I d$, which always corresponds to an element of torsion.

\section{Diagonal structure of GHW manifolds}

To start with, we present an important structural property of the class of GHW manifolds.

Theorem 3.1 The fundamental group of a Generalized Hantzsche-Wendt manifold is diagonal.

For the proof we start with two lemmas.

Lemma 3.2 Let $\rho: \mathbb{Z}_{2}^{n-1} \rightarrow \operatorname{GL}(n, \mathbb{Z})$ be a diagonal faithful integral representation with $-I d \notin \operatorname{Im}(\rho)$. Then there is $g \in \mathbb{Z}_{2}^{n-1}$ such that $\rho(g)=$ $\operatorname{diag}(-1, \ldots,-1,1,-1, \ldots,-1)$. Moreover, if furthermore $\operatorname{Im}(\rho) \nsubseteq \operatorname{SL}(n, \mathbb{Z})$ then there is $g \in \mathbb{Z}_{2}^{n-1}$ such that $\rho(g)=\operatorname{diag}(1, \ldots, 1,-1,1, \ldots, 1)$.

Proof. First, we regard $\mathrm{D}(n), \mathrm{D}^{+}(n)$ and $\operatorname{Im}(\rho)$ as $\mathbb{Z}_{2}$-vector spaces, of dimensions $n, n-1$ and $n-1$ respectively. Also, $\mathrm{D}(n)=\operatorname{Im}(\rho) \cup(-I d) \operatorname{Im}(\rho)$. Since the $C_{i}$ 's, for $1 \leq i \leq n$, are linearly independent, they cannot lie all in $\operatorname{Im}(\rho)$ simultaneously, thus there is at least one of them in $(-I d) \operatorname{Im}(\rho)$, or equivalently, there is a $-C_{i}$ in $\operatorname{Im}(\rho)$, as claimed.

The last assertion in the statement follows from a similar argument.

Lemma 3.3 Let $\Gamma$ be a $n$-rank Bieberbach group with translation lattice $\Lambda$. Suppose that $(B, b) \in \Gamma$ and $B$ has eigenvalues 1 , -1 , with corresponding eigenspaces $V^{+}$and $V^{-}$of dimensions 1 and $n-1$ respectively. Then $\Lambda=$ $\left(\Lambda \cap V^{+}\right) \oplus\left(\Lambda \cap V^{-}\right)$, and the orthogonal projection of $b$ onto $V^{+}$lies in $\frac{1}{2}\left(\Lambda \cap V^{+}\right) \backslash\left(\Lambda \cap V^{+}\right)$ 
Proof. We have

$$
\Gamma \ni(B, b+\lambda)^{2}=(I d,(B+I d)(b+\lambda)), \text { for every } \lambda \in \Lambda .
$$

The torsion-free condition implies that $0 \neq(B+I d)(b+\lambda) \in \Lambda$, for every $\lambda \in \Lambda$. Now, $\frac{1}{2}(B \pm I d)$ is the orthogonal projection onto $V^{ \pm}$. If we suppose that for some $\lambda \in \Lambda, \frac{1}{2}(B+I d)(b+\lambda)=\mu \in \Lambda$, then $(B+I d)(b+\lambda-\mu)=$ $(B+I d)(b+\lambda)-(B+I d)(\mu)=2 \mu-2 \mu=0$, a contradiction. Hence, the orthogonal projection of $b$ (and also of $b+\lambda$ for every $\lambda \in \Lambda$ ) onto $V^{+}$ lies in $\frac{1}{2} \Lambda \backslash \Lambda$. The fact that $V^{+}$is one-dimensional now implies that the orthogonal projection of $\Lambda$ onto $V^{+}$is exactly $\Lambda \cap V^{+}$. Hence $\Lambda$ splits into the direct sum of two lattices, and the lemma follows.

Proof of Theorem 3.1. We proceed by induction on the dimension $n$. If $n$ is small, it is known that the theorem holds. Let $\rho$ be the integral representation of $\Gamma$ defined on $G=\mathbb{Z}_{2}^{n-1}$ and let $\Lambda \simeq \mathbb{Z}^{n}$ be the lattice of translations of $\Gamma$. The nature of the holonomy group $G$ implies that each $\rho_{\mathbb{Q}}(g)$ diagonalizes, and since they commute, they diagonalize simultaneously, i.e., there is a $\mathbb{Q}$-basis $\mathcal{B}$ of $\Lambda$ such that $\left[\rho_{\mathbb{Q}}(g)\right]_{\mathcal{B}}$ is diagonal for every $g \in G$ (and the elements in the diagonal are \pm 1 's). Then, Lemma 3.2 applied to these integral matrices implies that $\exists g_{0} \in \mathbb{Z}_{2}^{n-1}$ such that $\left[\rho_{\mathbb{Q}}\left(g_{0}\right)\right]_{\mathcal{B}}=\operatorname{diag}(1,-1, \ldots,-1)$ (after reordering $\mathcal{B})$. Hence, $\rho\left(g_{0}\right)$ has eigenvalues +1 and -1 with multiplicities 1 and $n-1$ respectively. Now we can a apply Lemma 3.3, and thus $\Lambda$ splits into a direct sum $\Lambda=\Lambda_{1} \oplus \Lambda_{2}$ where $\operatorname{dim} \Lambda_{1}=1$ and $\operatorname{dim} \Lambda_{2}=n-1$. Also $\operatorname{span}_{\mathbb{R}} \Lambda_{1}$ (resp. $\left.\operatorname{span}_{\mathbb{R}} \Lambda_{2}\right)$ is the eigenspace of $\rho\left(g_{0}\right)$ with eigenvalue 1 (resp. -1) and the orthogonal projection of the translational part corresponding to $\rho\left(g_{0}\right)$ lies in $\frac{1}{2} \Lambda_{1} \backslash \Lambda_{1}$. Since $\rho\left(g_{0}\right) \rho(g)=\rho(g) \rho\left(g_{0}\right)$ for every $g \in \mathbb{Z}_{2}^{n-1}$, we have that $\Lambda_{1}$ and $\Lambda_{2}$ are stable under the action of $\rho(g)$, thus $\Lambda=\Lambda_{1} \oplus \Lambda_{2}$ is a $\mathbb{Z}_{2}^{n-1}$-module decomposition. Hence we have that $\rho=\chi \oplus \rho^{\prime}$, where $\chi$ is a character and $\rho^{\prime}$ has rank $n-1$, both defined on the same domain as $\rho$. Thus, $\forall g \in \mathbb{Z}_{2}^{n-1}$

$$
\rho(g)=\begin{array}{|l|l|}
\hline \chi(g) & \\
\hline & \rho^{\prime}(g) \\
\hline
\end{array}
$$

and

$\rho\left(g_{0}\right)=$\begin{tabular}{|l|l|}
\hline 1 & \\
\hline & $-I d$ \\
\hline
\end{tabular}

We may conjugate $\Gamma$ by $(I d, \mu)$ with $\mu \in \frac{1}{4} \Lambda_{1}$ and obtain that $p_{1}(b) \in \frac{1}{2} \Lambda_{1}$ for every $(B, b) \in \Gamma$, where $p_{1}$ is the orthogonal projection onto $\operatorname{span}_{\mathbb{R}} \Lambda_{1}$ (cf. [16, Lemma 1.4]). We define a character $\psi: \Gamma / \Lambda \simeq \mathbb{Z}_{2}^{n-1} \longrightarrow \mathbb{Z}_{2}=\{0,1\}$ by

$$
\psi((B, b))= \begin{cases}0 & \text { if } p_{1}(b) \in \Lambda_{1} \\ 1 & \text { if } p_{1}(b) \in \frac{1}{2} \Lambda_{1} \backslash \Lambda_{1} .\end{cases}
$$

It is well defined since all the elements $(B, b)$ in the same class in $\Gamma / \Lambda$ have the same $p_{1}(b) \bmod \Lambda_{1}$. 
Now we consider

$$
\Gamma \stackrel{r}{\longrightarrow} \Gamma / \Lambda \stackrel{\psi}{\longrightarrow} \mathbb{Z}_{2},
$$

where $r$ is the canonical projection. Set $\Gamma^{\prime}:=\operatorname{ker}(\psi \circ r)$. It is a subgroup of $\Gamma$ of index 2 and its elements $(B, b)$ have the property that $p_{1}(b) \equiv 0\left(\bmod \Lambda_{1}\right)$. The last fact shows that if we take $\Gamma^{\prime \prime}:=\left.\Gamma^{\prime}\right|_{\Lambda_{2}}$ (i.e. the restriction to the last $(n-1)$ coordinates of $B$ 's and $b$ 's), it is still a Bieberbach group, it has rank $n-1$ and holonomy group isomorphic to $\mathbb{Z}_{2}^{n-2}$. (Alternatively, we could have considered the subgroup $\Gamma_{0}:=\left\{(B, b) \in \Gamma: b_{1}=0\right\}$, where $b=\left(b_{1}, b_{2}\right) \in \Lambda_{1} \oplus \Lambda_{2}$, and observed that the restriction of $\Gamma_{0}$ to $\operatorname{span}_{\mathbb{R}} \Lambda_{2}$ gives an isomorphism between $\Gamma_{0}$ and $\Gamma^{\prime \prime}$.) Then we can apply induction to $\Gamma^{\prime \prime}$, hence it is diagonal. By the relation between $\Gamma$ and $\Gamma^{\prime \prime}$ it is clear that therefore $\Gamma$ is diagonal.

In the proof, the group denoted by $\Gamma^{\prime \prime}$ is a subgroup of $\Gamma$ (in the abstract sense, i.e., there is a monomorphism from $\Gamma^{\prime \prime}$ into $\Gamma$ ). This gives a way of going from one dimension to the previous one within the class of GHW manifolds, which will be used in Section 8. In other words we have

Corollary 3.4 Every GHW group has a subgroup isomorphic to a $G H W$ group of one lower rank.

In the oriented case, the existence of these manifolds in each odd dimension was proved in [13] and one realization in each odd dimension was shown in [17]. In [14] the oriented flat manifolds with (orthonormal) diagonal holonomy and holonomy group $\mathbb{Z}_{2}^{n-1}$ were studied and they were parametrized by certain directed graphs; also lower bounds for their cardinality were shown, growing rapidly with $n$.

Remark 3.5 (i) A direct consequence of Theorem 3.1 is that in the oriented case, the above definition of Generalized Hantzsche-Wendt manifolds coincides with the Hantzsche-Wendt manifolds considered in [14].

(ii) Generalized Hantzsche-Wendt manifolds are of minimal dimension among those with holonomy group $\mathbb{Z}_{2}^{k}, k$ fixed and $\geq 1$ (cf. [8]). Furthermore they are the only existing closed Riemannian $n$-manifolds with holonomy group $\mathbb{Z}_{2}^{n-1}$, since manifolds with finite holonomy group must be flat, by a theorem of Cartan-Ambrose-Singer (see [21, Cor. 3.4.7]).

(iii) For every $k, 1<k<n-1$, there are flat manifolds with holonomy group $\mathbb{Z}_{2}^{k}$ not of diagonal type. Examples of this situation are easy to construct (see end of Section 6).

(iv) Somehow opposite to diagonal manifolds are flat manifolds having indecomposable holonomy representation. We recall that there are many of them, and for example, for holonomy group $\mathbb{Z}_{2}^{2}$, they have been classified and the set of their dimensions is unbounded (cf. [1]). 


\section{Non-oriented GHW flat manifolds}

The class of non-oriented GHW manifolds includes two subclasses, depending on whether the first Betti number $\beta_{1}=0$ or 1 , or equivalently (cf. [9]), the classes of Bieberbach groups with trivial or non-trivial center.

Let us consider first the case with non-trivial center. From Theorem 3.1 and a similar observation as at the end of Section 2 for HW manifolds, this subclass have the integral holonomy representation uniquely determined. A simple example is the following (cf. [12]). Let $K_{n}$ be the subgroup of $\mathrm{E}(n)$ generated by the set $\left\{\left(C_{i}, s\left(C_{i}\right)\right): 0 \leq i \leq n-1\right\}$, where

$$
C_{0}=I \quad \text { and } \quad C_{i}=\operatorname{diag}(1, \ldots, 1, \underbrace{-1}_{i}, 1, \ldots, 1)
$$

are the $n \times n$ orthogonal matrices defined in (2.2). Moreover $s\left(C_{0}\right)=e_{1}$ and $s\left(C_{i}\right)=e_{i+1} / 2$ for other $i$. Here $e_{1}, e_{2}, \ldots, e_{n}$ denote the canonical basis of $\mathbb{Z}^{n}$. In [12] it was proved that $K_{n}$ is a Bieberbach group and that there exists the following short exact sequence of groups

$$
0 \longrightarrow K_{n-1} \longrightarrow K_{n} \longrightarrow \mathbb{Z} \longrightarrow 0,
$$

where the epimorphism $K_{n} \rightarrow \mathbb{Z}$ is given by the composition

$$
K_{n} \rightarrow K_{n} /\left[K_{n}, K_{n}\right] \rightarrow \mathbb{Z}
$$

However in general we have the following proposition.

Proposition 4.1 Let $\Gamma$ be a Generalized Hantzsche-Wendt group of dimension $n$ with non-trivial center. Then the kernel of the composition $f: \Gamma \rightarrow \Gamma /[\Gamma, \Gamma] \rightarrow \mathbb{Z}$ can be an oriented or a non-oriented Generalized Hantzsche-Wendt group.

Proof. First, it is not difficult to observe that ker $f$ is a Bieberbach group (cf. [2]). From the assumptions, the abelian group $\Gamma /[\Gamma, \Gamma] \simeq \mathbb{Z} \oplus F$ where $F$ is a finite group (cf. [9]). Let $f((A, a)) \in \mathbb{Z}$ be a generator for some $(A, a) \in$ $\Gamma$. We can assume, by using Lemma 3.2, that $A$ has eigenvalues $1,-1$, with corresponding eigenspaces $V^{+}$and $V^{-}$of dimensions 1 and $n-1$ respectively. Moreover we have an isomorphism $\operatorname{ker} f /(\Lambda \cap \operatorname{ker} f) \simeq \operatorname{ker} f /\left(\Lambda \cap V^{-}\right) \simeq$ $\mathbb{Z}_{2}^{n-2}$, where $\Lambda$ is the translation lattice of $\Gamma$. We have to show that $\mathbb{Z}_{2}^{n-2}$ is the holonomy group of the Bieberbach group ker $f$. But this follows from the assumptions and Lemma 3.3. To finish the proof we observe that for odd $n$, every ker $f$ is non-oriented. On the other hand, using semidirect product $\Gamma^{\prime} \rtimes_{\alpha} \mathbb{Z}$ with the integers, for any HW group $\Gamma^{\prime}$ of dimension $n$, we can define a $(n+1)$-rank GHW group with non-trivial center such that the kernel of the map onto $\mathbb{Z}$ is isomorphic to $\Gamma^{\prime}$. Here $\alpha: \mathbb{Z} \rightarrow \operatorname{Out}\left(\Gamma^{\prime}\right)$ is a homomorphism which send 1 to $-I d \in \operatorname{Out}\left(\Gamma^{\prime}\right)$. 
As mentioned above, the holonomy representation of GHW manifolds with $\beta_{1}=1$ is always equivalent to that of the above example, hence all these manifolds have the same rational homology, namely

Theorem 4.2 Let $M$ be a Generalized Hantzsche-Wendt flat manifold of dimension $n$ with first Betti number one. Then

$$
H_{j}(M, \mathbb{Q}) \simeq \begin{cases}\mathbb{Q} & \text { if } j=0,1 \\ 0 & \text { otherwise }\end{cases}
$$

Proof. From the definition and assumptions we have a short exact sequence

$$
0 \longrightarrow \mathbb{Z}^{n} \longrightarrow \pi_{1}(M) \longrightarrow \mathbb{Z}_{2}^{n-1} \longrightarrow 0
$$

where the action of $\mathbb{Z}_{2}^{n-1}$ on $\mathbb{Z}^{n}$ is given by the matrices $C_{i}, 1 \leq i \leq n-1$. This defines a representation $\rho: \mathbb{Z}_{2}^{n-1} \rightarrow \operatorname{GL}(n, \mathbb{Z})$. Using a similar method as in [17] we have a character $\Phi$ of $\rho$ given by the formula

$$
\Phi=\sum_{i=1}^{n} \chi_{i}
$$

where

$$
\chi_{i}=\chi_{0}^{\prime} \ldots \chi_{0}^{\prime} \chi_{1}^{\prime} \chi_{0}^{\prime} \ldots \chi_{0}^{\prime}, \quad 1 \leq i \leq n-1
$$

with $\chi_{1}^{\prime}$ the $i$-th of the $(n-1)$ factors in the product, and $\chi_{n}=\left(\chi_{0}^{\prime}\right)^{n-1}$. Here $\chi_{0}^{\prime}, \chi_{1}^{\prime}$ denote respectively a trivial and a non-trivial character of the involution group $\mathbb{Z}_{2}$. From [17]

$$
H_{j}(M, \mathbb{Q}) \simeq\left[\Lambda^{j}\left(\mathbb{Q}^{n}\right)\right]^{\mathbb{Z}_{2}^{n-1}}
$$

From its definition, the representation of $\mathbb{Z}_{2}^{n-1}$ on $\Lambda^{j}\left(\mathbb{Q}^{n}\right)$ has the following character

$$
\Lambda^{j}(\rho)=\sum_{1 \leq i_{1}<i_{2}<\cdots<i_{j} \leq n} \chi_{i_{1}} \chi_{i_{2}} \ldots \chi_{i_{j}}
$$

We have

$$
\Lambda^{j}(\rho)=\chi_{1} \sum_{2 \leq i_{2}<\cdots<i_{j} \leq n} \chi_{i_{2}} \ldots \chi_{i_{j}}+\sum_{2 \leq i_{1}<i_{2}<\cdots<i_{j} \leq n} \chi_{i_{1}} \chi_{i_{2}} \cdots \chi_{i_{j}} .
$$

Hence by induction and an immediate calculation the result follows.

According to [3] the number of isomorphism classes of GHW manifolds and their holonomy representations in dimensions up to 6 are as in the following table. 


\begin{tabular}{|c|c|c|c|c|c|c|}
\hline \multirow[b]{2}{*}{$\operatorname{dim}$} & \multicolumn{5}{|c|}{ number of GHW manifolds. } & \multirow{2}{*}{$\begin{array}{l}\text { number of } \\
\text { holon. repr. }\end{array}$} \\
\hline & $\beta_{1}=0$ & $\beta_{1}=1$ & total & orient. & non-orient. & \\
\hline 2 & 0 & 1 & 1 & 0 & 1 & 1 \\
\hline 3 & 1 & 2 & 3 & 1 & 2 & 2 \\
\hline 4 & 2 & 10 & 12 & 0 & 12 & 2 \\
\hline 5 & 23 & 100 & 123 & 2 & 121 & 3 \\
\hline 6 & 352 & 2184 & 2536 & 0 & 2536 & 3 \\
\hline
\end{tabular}

There are $62 \mathrm{HW}$ manifolds in dimension 7 and more that 1000 in dimension 9 (cf. [15]).

\section{Holonomy representations}

If we consider the case of non-oriented Generalized Hantzsche-Wendt Bieberbach groups with trivial center then we have many more possibilities for the holonomy representation. Let us start establishing the following general result.

Theorem 5.1 Let $\rho: \mathbb{Z}_{2}^{k} \rightarrow \operatorname{GL}(n, \mathbb{Z})$ be a diagonal faithful integral representation with $-I d \notin \operatorname{Im}(\rho)$ and $k<n$. Then there is a Bieberbach group whose integral holonomy representation is $\rho$.

Proof. It suffices to prove the case $k=n-1$, since in the cases $k<n-1$ we can use the lemma below to induce from $\rho$ a new representation defined on $\mathbb{Z}_{2}^{n-1}$; then apply the result for $n-1$ to obtain a diagonal Bieberbach group $\Gamma$ with the new integral holonomy representation; and finally, choose the appropriate proper subgroup of $\Gamma$ of index $2^{n-1-k}$ corresponding to the original integral representation $\rho$. Note that this chosen subgroup has the same lattice of translations as $\Gamma$, and by construction, it is always a diagonal Bieberbach group.

Lemma 5.2 If $\sigma: \mathbb{Z}_{2}^{k} \rightarrow \operatorname{GL}(n, \mathbb{Z})$ is a diagonal faithful integral representation with $-I d \notin \operatorname{Im}(\sigma)$ and $k<n-1$, then there exists an integral representation $\tau: \mathbb{Z}_{2}^{k+1} \rightarrow \mathrm{GL}(n, \mathbb{Z})$ with the same properties as $\sigma$ and containing properly $\sigma$, i.e., $\tau \uparrow_{\mathbb{Z}_{2}^{k} \times\{0\}}=\sigma$.

Proof. Denote $\sigma^{-}:=-I d \circ \sigma$, i.e. $\sigma^{-}(g):=-(\sigma(g))$. Since $-I d \notin \operatorname{Im}(\sigma)$, we have $\operatorname{Im}(\sigma) \cap \operatorname{Im}\left(\sigma^{-}\right)=\emptyset$. Since $2 \times 2^{k}<2^{n}$, there is $B \in \mathrm{D}(n) \backslash$ $\left(\operatorname{Im}(\sigma) \cap \operatorname{Im}\left(\sigma^{-}\right)\right)$. Now define $\tau: \mathbb{Z}_{2}^{k+1} \rightarrow \mathrm{D}(n)$ as the unique representation satisfying $\tau \uparrow_{\mathbb{Z}_{2}^{k} \times\{0\}}=\sigma$ and $\tau \uparrow_{\{0\}^{k} \times \mathbb{Z}_{2}}=\langle B\rangle$ (here we are identifying $\langle B\rangle$ with the representation of $\mathbb{Z}_{2}$ in $\mathbb{Z}^{n}$ whose nontrivial element acts by $B$ ). Since $B \notin \operatorname{Im}(\sigma), \tau$ is a faithful representation; since $B \notin \operatorname{Im}\left(\sigma^{-}\right),-I d \notin \operatorname{Im}(\tau)$, and therefore $\tau$ has the desired properties. 
(Continuation of the proof of Theorem 5.1) We continue the proof by induction on $n$. If $n=2,3$, it is easy to prove it. If $\operatorname{Im}(\rho) \subseteq \operatorname{SL}(n, \mathbb{Z})$, then $n$ must be odd and we know that there are manifolds with $\rho$ as its integral representation, namely the Hantzsche-Went manifolds as in [13], [14], [17]. Otherwise $\operatorname{Im}(\rho)$ contains elements of determinant -1 , then by Lemma 3.2, there is $C_{i} \in \operatorname{Im}(\rho)$, that after reordering the coordinates we may assume to be $C_{1}=\operatorname{diag}(-1,1, \ldots, 1)$. Denote $p_{1}$ the projection onto the first coordinate and $p_{r}$ the projection onto the last $n-1$ coordinates. Next, consider $\operatorname{ker}\left(p_{1} \circ \rho\right)$, which is a subgroup of $\mathbb{Z}_{2}^{n-1}$ of index 2. Denote the inclusion $\operatorname{ker}\left(p_{1} \circ \rho\right) \stackrel{\iota}{\hookrightarrow} \mathbb{Z}_{2}^{n-1}$, and define $\rho^{\prime}:=p_{r} \circ \rho \circ \iota$. Then $\rho^{\prime}$ is faithful, diagonal and $-I d \notin \operatorname{Im}\left(\rho^{\prime}\right)$, however it is of rank $n-1$ and defined from a group isomorphic to $\mathbb{Z}_{2}^{n-2}$. By induction, there is a Bieberbach group $\Gamma^{\prime}$ whose integral holonomy representation is $\rho^{\prime}$. Moreover, we may assume that all the elements in $\Gamma^{\prime}$ are of the form $\left(B^{\prime}, b^{\prime}\right)$ with $B^{\prime} \in \operatorname{Im}\left(\rho^{\prime}\right)$ and $b^{\prime} \in \frac{1}{2} \mathbb{Z}^{n-1}$ (see Lemma 1.4 in $[16]$ ).

To complete the proof one might use previous known results, as in [18], but we prefer the following explicit method, since it is elementary and provides a method which will be useful in Section 8 .

\begin{tabular}{|ccc|c|ccc|}
\hline 1 & $\cdots$ & 1 & -1 & -1 & $\cdots$ & -1 \\
\hline & & & 1 & & & \\
& & & $\vdots$ & & $\Gamma^{\prime}$ & \\
& $\Gamma^{\prime}$ & & 1 & & & \\
& & & 1 & & & \\
\hline
\end{tabular}

The previous picture gives an idea of the Bieberbach group we are constructing. In the middle column is placed $C_{1}$. We need a vector $c \in \mathbb{R}^{n}$ to form a torsion-free element $\left(C_{1}, c\right)$. We choose $c=e_{2} / 2$ (any other choice $c=e_{i} / 2$ for $i>2$ would work well too). Now we define new elements by using the elements in $\Gamma^{\prime}$ in the following way: if $\left(B^{\prime}, b^{\prime}\right) \in \Gamma^{\prime}$, then we define $(B, b)$ by $B:=\left[\begin{array}{ll}1 & \\ & B^{\prime}\end{array}\right]$ and $b:=\left(b_{1}^{\prime}, 0, b_{2}^{\prime}, \ldots, b_{n-1}^{\prime}\right)$, where $b_{i}^{\prime}, 1 \leq i \leq n$, are the coordinates of $b^{\prime}$. Then $\widetilde{\Gamma^{\prime}}:=\left\langle(B, b):\left(B^{\prime}, b^{\prime}\right) \in \Gamma^{\prime} ; L_{e_{i}}: 1 \leq i \leq n\right\rangle$ is an $n$-rank Bieberbach group with holonomy group $\operatorname{ker}\left(p_{1} \circ \rho\right)$ (it is torsionfree since $\Gamma^{\prime}$ is so). We claim that $\left\langle(B, b) \in \widetilde{\Gamma^{\prime}},\left(C_{1}, c\right)\right\rangle$ is a Bieberbach group with integral holonomy representation $\rho$. It is clear that its integral representation is $\rho$, so the only point to be explained is that it is torsionfree. This condition is clear for elements in $\widetilde{\Gamma^{\prime}}$ and for $\left(C_{1}, c\right)$. Now the products $(B, b)\left(C_{1}, c\right)$ are of the forms $(D, d)$ with $D=\left[\begin{array}{cc}-1 & 0 \\ 0 & B^{\prime}\end{array}\right]$ and $d=\left(*, \frac{1}{2}, b_{2}^{\prime}, \ldots, b_{n-1}^{\prime}\right)$. Since $\Gamma^{\prime}$ is torsion-free, then the elements $(D, d)$ are torsion-free too. 
Now we are interested in the holonomy representations of GHW groups.

Theorem 5.3 The total number of inequivalent integral holonomy representations occurring in Generalized Hantzsche-Wendt Bieberbach groups of dimension $n$ equals $n / 2$ for $n$ even and $(n+1) / 2$ for $n$ odd.

Proof. By definition, each holonomy representation is defined by a hyperplane in $\mathbb{Z}_{2}^{n}$. From linear algebra, it is the kernel of a linear map with matrix $\left[x_{1}, x_{2}, \ldots, x_{n}\right]$, where $x_{i} \in \mathbb{Z}_{2}=\{0,1\}$. Hence, we have the following $n$ classes of diagonal representations:

$$
[1,0, \ldots, 0],[1,1,0, \ldots, 0], \ldots,[1,1, \ldots, 1,0],[1,1, \ldots, 1] .
$$

Since we are considering torsion-free groups, all the hyperplanes corresponding to holonomy representations do not have the vector $[1,1, \ldots, 1]$, since $-I d$ corresponds to an element of torsion. Hence we have to consider only vectors with an odd number of nonzero elements. We claim that diagonal holonomy representations defined by such vectors are all inequivalent. In fact, let us consider a hyperplane (representation) as the set of $n \times n$ diagonal matrices. It suffices to count the number of matrices $A$ such that the group of fix points $\left(\mathbb{Z}^{n}\right)^{A}$ of the action of $A$ is isomorphic to the integers $\mathbb{Z}$, i.e. matrices of the form $A=\operatorname{diag}(-1, \ldots,-1,1,-1, \ldots,-1)$. It is not difficult to see that for different hyperplanes, the number of such $A$ 's is different. Summing up we have for $n$ even, $n / 2$ and for $n$ odd, $(n+1) / 2$ possibilities, which indeed occur by Theorem 5.1.

As a consequence, the number of integral representations occurring in non-oriented GHW groups with trivial center is $(n / 2)-1$ for $n$ even and $((n+1) / 2)-2$ for $n$ odd.

\section{Examples}

In this section we present an explicit example of GHW groups with trivial center in each dimension $n$. In the notation of the proof of Theorem 5.3, its holonomy representation corresponds to hyperplanes $[1,1, \ldots, 1,1]$ in the odd case and to $[1,1, \ldots, 1,1,0]$ in the even case. For $n \geq 2$, let $\Gamma_{n}$ be the subgroup of $\mathrm{E}(n)$ generated by the set $\left\{\left(B_{i}, s\left(B_{i}\right)=x_{i}\right): 1 \leq i \leq n-1\right\}$. Here $B_{i}$ 's are the $n \times n$ orthogonal matrices:

$$
B_{i}:=-C_{i}=\operatorname{diag}(-1, \ldots,-1, \underbrace{1}_{i},-1, \ldots,-1)
$$

and

$$
x_{i}=s\left(B_{i}\right)=e_{i} / 2+e_{i+1} / 2 \text { for } 1 \leq i \leq n-1 .
$$


These groups have trivial center for $n \geq 3$. In [17] (see also [14]) it was proved that $\Gamma_{n}$ are Bieberbach groups for odd $n$. For even $n$ the groups $\Gamma_{n}$ are also Bieberbach groups (one might also use Proposition 2.1 in [5] to prove it) in particular because we have

Proposition 6.1 For $n \geq 2$, there is a monomorphism $\phi_{n}: \Gamma_{n} \rightarrow \Gamma_{n+1}$ of groups. Moreover $\phi_{n}\left(\Gamma_{n}\right)$ is not a normal subgroup of $\Gamma_{n+1}$.

Proof. Let $\left\{\left(B_{i}, x_{i}\right): 1 \leq i \leq n-1\right\}$ be a set of generators of $\Gamma_{n}$. We have an inclusion $k: \mathbb{Z}^{n} \rightarrow \mathbb{Z}^{n+1}$, where $b \in \mathbb{Z}^{n}$ is mapped to $k(b)=(b, 0) \in \mathbb{Z}^{n+1}$. Next, we consider the $(n+1) \times(n+1)$ matrices $\bar{B}_{i}:=\left[\begin{array}{c|c}B_{i} & 0 \\ \hline 0 & -1\end{array}\right], 1 \leq i \leq n$. We define

$$
\phi_{n}\left(\left(B_{i}, x_{i}\right)\right)=\left(\bar{B}_{i}, k\left(x_{i}\right)\right) .
$$

Finally, it suffices to conjugate by the translation $(I d,(0, \ldots, 0,1))$ an element $\phi_{n}\left(\left(B_{1},(1 / 2,1 / 2,0, \ldots, 0)\right)\right.$ to show that the image of $\Gamma_{n}$ is not a normal subgroup.

By analogy with [17] we have.

Theorem 6.2 For $n \geq 2$,

$$
H_{j}\left(\mathbb{R}^{n} / \Gamma_{n}, \mathbb{Q}\right) \simeq \begin{cases}\mathbb{Q} & \text { if } j=0 \\ \mathbb{Q} & \text { if } n \text { is even and } j=n-1 \\ \mathbb{Q} & \text { if } n \text { is odd and } j=n \\ 0 & \text { otherwise }\end{cases}
$$

Proof. For odd $n$ it was proved in [17]. The proof for $n=2 k$ follows the same structure as the proof of Theorem 4.2. We have just to replace $n$ by $2 k, M$ by $\Gamma_{2 k-1}$, and the action of matrices $C_{i}$ by the action of $B_{i}$ as in (6.1), thus the characters change accordingly. Now from the equivalent to formula (4.2), an immediate calculation shows that no summand is trivial there, then $\operatorname{dim}_{\mathbb{Q}}\left[\Lambda^{j}\left(\mathbb{Q}^{2 k}\right)\right]^{\mathbb{Z}_{2}^{2 k-1}}=0$ for each $j, 1 \leq j \leq 2 k-2$. Moreover

$$
\begin{aligned}
& \sum_{g \in \mathbb{Z}_{2}^{2 k-1}} \frac{1}{2^{2 k-1}} \sum_{1 \leq i_{1}<i_{2}<\cdots<i_{2 k-1} \leq 2 k} \chi_{i_{1}} \chi_{i_{2}} \ldots \chi_{i_{j}}(g)= \\
& =\sum_{g \in \mathbb{Z}_{2}^{2 k-1}} \frac{1}{2^{2 k-1}} \sum_{i=1}^{2 k} \chi_{1} \ldots \hat{\chi}_{i} \ldots \chi_{2 k}(g)=\frac{1}{2^{2 k-1}} \sum_{i=0}^{2 k-1}(2 k-2 i)\left(\begin{array}{c}
2 k-1 \\
i
\end{array}\right)=1 .
\end{aligned}
$$

Here $\chi_{1} \ldots \hat{\chi}_{i} \ldots \chi_{2 k}$ denotes the product of characters $\chi_{1} \ldots \chi_{i} \ldots \chi_{2 k}$ without the character $\chi_{i}$. Hence $\operatorname{dim}_{\mathbb{Q}}\left[\Lambda^{2 k-1}\left(\mathbb{Q}^{2 k}\right)\right]^{\mathbb{Z}_{2}^{2 k-1}}=1$. 
Finally, one can calculate that

$$
\sum_{g \in \mathbb{Z}_{2}^{2 k-1}} \chi_{1} \chi_{2} \ldots \chi_{2 k}(g)=\sum_{i=0}^{2 k-1}(-1)^{i}\left(\begin{array}{c}
2 k-1 \\
i
\end{array}\right)=0 .
$$

In the formulas above we use the definition of characters on the elements of $\mathbb{Z}_{2}^{2 k-1}$ considered as elements of $\mathbb{Z}_{2}$-vector space.

A small modification to the groups $K_{n}$ defined in Section 4 produces examples as required in Remark 3.5(iii): we replace the $(1,1)$ entries in matrices $C_{i}$ by $2 \times 2$ matrices of the form

$$
J:=\left[\begin{array}{ll}
0 & 1 \\
1 & 0
\end{array}\right] \text { if the entry is a }-1
$$

and

$$
\text { the } 2 \times 2 \text { identity }\left[\begin{array}{ll}
1 & 0 \\
0 & 1
\end{array}\right] \text { if the entry is a } 1 \text {. }
$$

Now the dimension increases by one, while the holonomy group remains the same. For example, for $n=2$, we had for $K_{2}$ (the Klein bottle group), matrix $C_{1}=\left[\begin{array}{cc}-1 & 0 \\ 0 & 1\end{array}\right]$ and vector $s\left(C_{1}\right)=\frac{e_{2}}{2}$; and now we have a $3 \times 3$ matrix $\left[\begin{array}{ll}J & 0 \\ 0 & 1\end{array}\right]$ and vector $\frac{e_{3}}{2}$ to obtain a 3-dimensional Bieberbach group which is not of diagonal type.

Hence these are, as claimed, non-diagonal examples in the case that $k+2$ equals the dimension (where $\mathbb{Z}_{2}^{k}$ is the holonomy group). The verification that these are indeed Bieberbach groups is easy, and analogous to that for $K_{n}$. The smaller the $k$ (with fixed dimension), the easier the construction of these examples.

\section{Outer automorphism groups}

In this section we give an estimate for the order of the outer automorphism group of GHW Bieberbach groups. In the case of the groups $K_{n}$ considered in Section 4, we prove that the group $\operatorname{Aut}\left(K_{n}\right) / \operatorname{Inn}\left(K_{n}\right)=\operatorname{Out}\left(K_{n}\right) \simeq \mathbb{Z}_{2}^{n}$. We denote by $|A|$ the order of a group $A$.

Theorem 7.1 Let $\Gamma$ be a Generalized Hantzsche-Wendt group of dimension $n$. Then we have

$$
|\operatorname{Out}(\Gamma)| \leq 2^{n+1} n !
$$

Moreover in the case with non-trivial center we have

$$
|\operatorname{Out}(\Gamma)| \leq 2^{n}(n-1) ! \text {. }
$$


Proof. Let $N$ denote the normalizer in $\operatorname{GL}(n, \mathbb{Z})$ of the holonomy group $\mathbb{Z}_{2}^{n-1}$. Then $N$ acts in a natural way on $H^{2}\left(\mathbb{Z}_{2}^{n-1}, \mathbb{Z}^{n}\right)$.

Let $\alpha \in H^{2}\left(\mathbb{Z}_{2}^{n-1}, \mathbb{Z}^{n}\right)$ denote the cohomology class giving rise to the group $\Gamma$, and let $N_{\alpha}$ denote its stabilizer in $N$. Then $\mathbb{Z}_{2}^{n-1}$ is a normal subgroup of $N_{\alpha}$ and we have a short exact sequence (cf. [2], [10])

$$
0 \longrightarrow H^{1}\left(\mathbb{Z}_{2}^{n-1}, \mathbb{Z}^{n}\right) \longrightarrow \operatorname{Out}(\Gamma) \longrightarrow N_{\alpha} / \mathbb{Z}_{2}^{n-1} \longrightarrow 0 .
$$

From [10] for HW groups, $N=S_{n} \imath \mathbb{Z}_{2}$ is semidirect product of the symmetric group $S_{n}$ and $\mathbb{Z}_{2}^{n}$. For a non-oriented GHW group with non-trivial center, $N=\mathbb{Z}_{2} \times\left(S_{n-1} \prec \mathbb{Z}_{2}\right)$. In fact, the normalizer $N \simeq \mathbb{Z}_{2} \oplus N_{1}$ because the image of the holonomy representation contains a unique element having as its diagonal entries only one 1 and the remaining are all -1 's, (see Lemma 3.2). Moreover $N_{1} \simeq S_{n-1} \prec \mathbb{Z}_{2}$. In the case of non-oriented $\Gamma$ with trivial center we can write the diagonal group $\mathbb{Z}_{2}^{n}$ in the group $\operatorname{GL}(n, \mathbb{Z})$ as a sum of a holonomy group $\mathbb{Z}_{2}^{n-1}$ and a set $(-I d) \mathbb{Z}_{2}^{n-1}$. Hence $N_{\mathrm{GL}(n, \mathbb{Z})}\left(\mathbb{Z}_{2}^{n-1}\right) \subset$ $N_{\mathrm{GL}(n, \mathbb{Z})}\left(\mathbb{Z}_{2}^{n}\right) \simeq S_{n}\left(\mathbb{Z}_{2}\right.$. From (7.1) we only have to prove that $\left|H^{1}\left(\mathbb{Z}_{2}^{n-1}, \mathbb{Z}^{n}\right)\right|$ equals $2^{n}$ in the case of trivial center and $2^{n-1}$ in case of non-trivial center. But this is proved in [2].

Corollary 7.2 For $n \geq 2$, $\operatorname{Out}\left(K_{n}\right) \simeq \mathbb{Z}_{2}^{n}$.

Proof. From above the normalizer $N$ of the holonomy representation is equal to $\mathbb{Z}_{2} \times S_{n-1} \prec \mathbb{Z}_{2}$, where the permutation group acts on the first $(n-1)$ coordinates. Let $s \in H^{2}\left(\mathbb{Z}_{2}^{n-1}, \mathbb{Z}^{n}\right)$ be a cocycle which corresponds to the group $K_{n}$ of Section 4 . Then $N_{s}=\mathbb{Z}_{2}^{n}$. In fact it is enough to observe that for any non-trivial $\sigma \in S_{n-1}, \sigma s \neq s$. We have

$$
(\sigma s)\left(B_{i}\right)=\sigma s\left(\sigma^{-1} B_{i} \sigma\right)=\sigma s\left(B_{\sigma^{-1}(i)}\right)
$$

for any generator $B_{i} \in \mathbb{Z}_{2}^{n-1}$ and $i=1,2, \ldots, n-1$, see [10]. We are looking for the permutation $\sigma \in S_{n-1}$ such that the cocycle $\sigma s-s$ is trivial. We have two cases. First assume that $\sigma^{-1}(i)+1<n$. Hence $\sigma^{-1}(i)+1=\sigma^{-1}(i+1)$. From $\sigma^{-1}(i)+1=n$ it follows that $i=n-1$. Summing up we have $\sigma \in N_{s}$ if and only if, for $i<(n-1), \sigma(i+1)=\sigma(i)+1$ and $\sigma(n-1)=n-1$. Hence for $i=n-2$ we have $\sigma(n-2)=n-2$ and by induction $\sigma$ is identity. To finish the proof we observe that $N_{s} / \mathbb{Z}_{2}^{n-1}=\mathbb{Z}_{2}$ and it is generated by the image of $-I d$. Now it is enough to apply the short exact sequence (7.1).

Remark 7.3 From [2], for GHW manifolds $M^{n}$ of dimension $n$ with first Betti number $\beta_{1}$, the group $\operatorname{Aff}\left(M^{n}\right) /\left(S^{1}\right)^{\beta_{1}}$ of affine diffeomorphisms of $M^{n}$ is isomorphic to the group $\operatorname{Out}\left(\pi_{1}\left(M^{n}\right)\right)$. 


\section{A graph connecting the GHW manifolds}

In this section we start the discussion of relations between the generalized Hantzsche-Wendt manifolds of different dimensions. For manifolds with first Betti number one we have relations from E. Calabi's construction (see Proposition 4.1 and [21], p. 125). But we have also a lot of links between GHW groups of different dimensions in the case when one of them has trivial center. We must say that this subject needs further research and we want to come back to it in the future. Most of the observations in this section are corollaries from previous ones, and we shall omit some proofs.

Let us start defining a graph $\mathbf{G}$ as follows:

- The vertices of $\mathbf{G}$ are the GHW groups (or manifolds).

- We say that a vertex has dimension $n$ if it corresponds to a GHW manifold of dimension $n$.

- Edges are defined only between vertices in consecutive dimensions.

- There is an edge between two vertices (in consecutive dimensions) if one of the corresponding GHW groups is a subgroup of the other (in an abstract sense, i.e. there is a monomorphism from one into the other).

From Corollary 3.4 we see that in $\mathbf{G}$ there is always (at least) an edge 'down' from each vertex. As a direct consequence we have

Corollary 8.1 (i) The fundamental group of the Klein bottle is a subgroup of every GHW group. (ii) The graph $\mathbf{G}$ is connected.

Moreover from Figure 1 we see that $\mathbf{G}$ is not a tree.

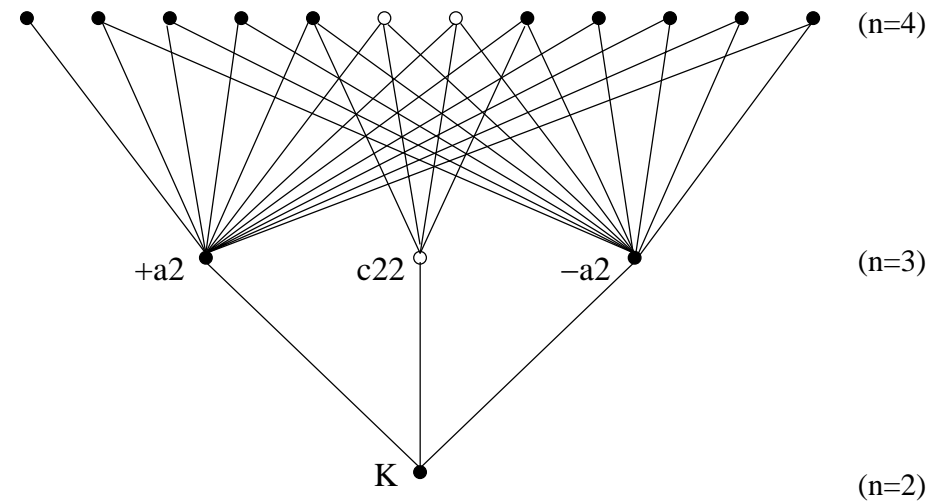

Figure 1: The graph of GHW manifolds up to $n=4$.

On the other hand, from the method in the proof of Theorem 5.1 it is possible to deduce that any GHW group in dimension $n$ is subgroup of a GHW group 
in dimension $n+1$. Moreover, we can prove that it is indeed subgroup of at least two different GHW groups in dimension $n+1$ (we omit the proof for brevity). In the graph $\mathbf{G}$ this means that from any vertex there are at least two edges 'up'.

In Figure 1, a point $\circ$ (resp. a bullet •) denotes a GHW manifold with first Betti number zero (resp. one); $K$ is the Klein bottle; $+a 2$ and $-a 2$ are the first and second amphidicosm respectively, they are the two nonoriented GHW manifolds of dimension 3; $c 22$ is the didicosm (or the oriented Hantzsche-Wendt 3-dimensional flat manifold). The new names are due to a proposal by John H. Conway to name the ten flat 3-manifolds according to their properties, which we adopt (this terminology is introduced in [4]).

Other edges in Figure 1 are obtained by extensions to the methods of theorems 3.1 and 5.1.

There are many other properties of $\mathbf{G}$ to be explored. For instance, a distance between vertices can be defined in the standard way for graphs.

In dimension 5 there are only two HW manifolds (cf. [14]). With the method of Theorem 3.1, it is not difficult to prove that they have both $\Gamma_{4}$ (defined in Section 6) as a subgroup, and therefore the distance between them is just two. We also have

Proposition 8.2 The fundamental group of the didicosm (or HantzscheWendt 3-manifold) is a subgroup of every GHW group with trivial center.

Proof. Denote by $\Gamma$ the given GHW group. By Lemma 3.2 there is $(B, b) \in \Gamma$ such that (after permuting coordinates) $B=\operatorname{diag}(1,-1, \ldots,-1)$ and $b=\left(\frac{1}{2}, *, \ldots, *\right)$, where each $*$ is in $\left\{0, \frac{1}{2}\right\}$. Since $\Gamma$ has trivial center there is another element $(C, c) \in \Gamma$ with $C=\operatorname{diag}(-1,1,-1, \pm 1, \ldots, \pm 1)$ and the second coordinate of $c$ equals $1 / 2$. Now the integral representation corresponding to $\langle B, C, B C\rangle$ is the direct sum of the three non-trivial characters of $\mathbb{Z}_{2} \oplus \mathbb{Z}_{2}$ (with certain multiplicities). Then it is not difficult to check that the subgroup of $\Gamma$ generated by $(B, b)$ and $(C, c)$ is isomorphic to the fundamental group of the didicosm.

In [6] the analogous result was proved for Bieberbach groups with holonomy group $\mathbb{Z}_{2} \oplus \mathbb{Z}_{2}$ (instead of GHW groups) and trivial center (see also [20]).

By (4.1) the groups $K_{n}, n \geq 2$, form an infinite chain in G. Also the groups $\Gamma_{n}, n \in \mathbb{N}, n \geq 3$ from Section 6 form an infinite chain by Proposition 6.1.

Finally, we remark that the edges in $\mathbf{G}$ sometimes correspond to normal subgroups and sometimes not (or they can correspond to both simultaneously). For instance, the chain obtained by Proposition 6.1 is not normal. Also, $\pi_{1}(K)$ can be injected as a normal and as a non-normal subgroup in $\pi_{1}(+a 2)$ and in $\pi_{1}(-a 2)$ but it is never a normal subgroup of $\pi_{1}(c 22)$. 


\section{Some problems and questions:}

1. Are there relations between Fibonacci groups and the fundamental groups of Hantzsche-Wendt manifolds? (cf. [19])

2. Are there other geometric structures on oriented GHW manifolds?

- Is it possible to introduce some invariants on them? (in analogy with three dimensional rational homology sphere as Casson-Walker and Seiberg-Witten invariants)

3. Is it possible to improve the upper bound given in Theorem 7.1? Could the factor $n$ ! be replaced by $n$ ?

4. What are the geometrical and topological properties of the 'graph' of GHW manifolds?

\section{References}

[1] Bovdi, V.A.; Gudivok, P. M.; Rudko, V. P.: Torsion-free groups with indecomposable holonomy group. I. J. Group Theory 5 (2002), no. 1, 75-96.

[2] Charlap, L.S.: Bieberbach Groups and Flat Manifolds. Universitext. Springer-Verlag, New York, 1986.

[3] Cid, C.; Schulz, T.: Computation of Five and Six dimensional Bieberbach groups. Experiment. Math. 10 (2001), no. 1, 109-115.

[4] Conway, J. H.; Rossetti, J. P.: Describing the Platycosms. Preprint. http://arxiv.org/abs/math.DG/0311476.

[5] Dotti Miatello, I; Miatello, R. J.: Isospectral compact flat manifolds. Duke Math. J. 68 (1992), no. 3, 489-498.

[6] Gupta, N.; Sidki, S.: On torsion-free metabelian groups with commutator quotients of prime exponent. Internat. J. Algebra Comput. 9 (1999), no. 5, 493-520.

[7] Hantzsche, W.; Wendt, H.: Dreidimensional euklidische Raumformen. Math. Ann. 110 (1934-35), 593-611.

[8] Hiller, H.; Marciniak, Z.; Sah, C. H.; Szczepański, A.: Holonomy of flat manifolds with $b_{1}=0$. II. Quart. J. Math. Oxford (2) 38 (1987), no. $150,213-220$.

[9] Hiller, H.; SAH, C. H.: Holonomy of flat manifolds with $b_{1}=0$. Quart. J. Math. Oxford (2) 37 (1986), no. 146, 177-187.

[10] Hiss, G.; Szczepański, A.: Flat manifolds with only finitely many affinities. Bull. Polish Acad. Sci. Math. 45 (1997), no. 4, 349-357.

[11] Kotschick, D.: On products of harmonic forms. Duke Math. J. 107 (2001), no. 3, 521-531.

[12] Lee, R.; Szczarba, R. H.: On the integral Pontrjagin classes of a Riemannian flat manifold. Geometriae Dedicata 3 (1974), 1-9. 
[13] Maxwell, G.: Compact Euclidean Space Forms. J. Algebra 44 (1977), no. $1,191-195$.

[14] Miatello, R. J.; Rossetti, J. P.: Isospectral Hantzsche-Wendt manifolds. J. Reine Angew. Math. 515 (1999), 1-23.

[15] Miatello, R. J.; Rossetti, J. P.: Hantzsche-Wendt manifolds of dimension 7. In Differential geometry and applications (Brno, 1998), 379-390. Masaryk Univ., Brno, 1999.

[16] Miatello, R. J.; Rossetti, J. P.: Comparison of Twisted P-Form Spectra for Flat Manifolds with Diagonal Holonomy. Ann. Global Anal. Geom. 21 (2002), no. 4, 341-376.

[17] Szczepański, A.: Aspherical manifolds with the $Q$-homology of a sphere. Mathematika 30 (1983), no. 2, 291-294.

[18] Szczepański, A.: Euclidean space forms with the first Betti number equal to zero. Quart. J. Math. Oxford (2) 36 (1985), no. 144, 489-493.

[19] Szczepański, A.: The Euclidean representations of the Fibonacci groups. Q. J. Math. 52 (2001), no. 3, 385-389.

[20] Tirao, P.: Primitive compact flat manifolds with holonomy group $Z_{2} \oplus Z_{2}$. Pacific J. Math. 198 (2001), no. 1, 207-233.

[21] Wolf, J.A.: Spaces of constant curvature. McGraw-Hill, New YorkLondon-Sydney, 1967.

Recibido: 25 de noviembre de 2003

$$
\begin{array}{r}
\text { Juan Pablo Rossetti } \\
\text { FaMAF(Ciem) } \\
\text { Universidad Nacional de Córdoba } \\
5000 \text { - Córdoba, Argentina } \\
\text { rossetti@mate. uncor.edu } \\
\text { Andrzej Szczepański } \\
\text { Institute of Mathematics } \\
\text { University of Gdańsk } \\
\text { ul.Wita Stwosza } 57 \\
80 \text { - 952 Gdańsk, Poland } \\
\text { matas@paula.univ.gda.pl }
\end{array}
$$

First author was supported by a Guggenheim Fellowship and Conicet. 